\title{
UNA NOTA SOBRE EL CONCEPTO \\ Y LA FUNDAMENTACIÓN DE LOS \\ DERECHOS HUMANOS. \\ (A PROPÓSITO DE LA POLÉMICA \\ SOBRE LOS DERECHOS MORALES)
}

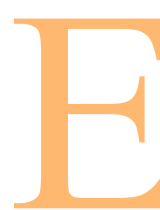

1 debate en torno a los derechos morales no es de ayer. No trato de resolverlo, pues, en unas pocas páginas (aunque hay quien entiende que pudiera ocupar tan sólo unas líneas: las necesarias para hacer ver que se trata sólo de un prejuicio lingüístico). En lo que sigue, intentaré únicamente mostrar, en primer lugar (I), la relación entre los problemas de concepto y fundamentación, de donde deriva la dificultad de abordarlos por separado. Ante esa dificultad caben dos planteamientos: optar por una solución monista (una misma respuesta a las cuestiones de concepto y fundamento) como lo hacen las teorías iusnaturalistas o iuspositivistas legalistas extremas, o aceptar que, pese a las dificultades, se impone distinguir esos dos problemas, y en ese caso adoptar una solución dualista. (II) En segundo lugar, y habida cuenta de los problemas a sortear por la solución dualista, y, asimismo, de las irresolubles dificultades de la respuesta iusnaturalista tout court, examinaré la tesis de los derechos morales en la medida en que parece constituir una versión depurada de la solución monista, desprovista de los problemas que afectan a la versión iusnaturalista. Tras ese examen, concluiré (III) que la teoría de los derechos morales tiene un alcance engañoso: (a) inicialmente se mantiene como tal solución monista, pero (b) en casi todos sus representantes aparece formulada de hecho como respuesta al problema conceptual: ¿qué son los derechos humanos? ¿qué significa tener derecho a X?, cuando en realidad (C) es tan sólo una teoría relativa al fundamento de los derechos y ni aún así constituye tal fundamento, pues se remite a otra instancia: las necesidades, los bienes básicos, los valores. Por supuesto, el alcance de esta 
reflexión es limitado: Una simple ojeada a la bibliografía existente sobre el concepto y fundamentación de los derechos humanos es suficiente para desanimar a quien pretenda formular aportaciones originales al respecto. Desde luego, no es esa la intención de estas líneas, que se proponen tan sólo una reflexión crítica sobre el status quaestionis, con especial referencia al debate doctrinal en nuestro país.

Para abordar con mayor concreción el problema, parece conveniente plantear con cierto detenimiento una reflexión previa, acerca de la posibilidad de dar respuesta con independencia absoluta a los problemas de concepto y fundamentación. En efecto, no es infrecuente que, cuando se formulan una y otra cuestión, se tienda a separarlas, manteniendo la primera, por así decirlo, en el terreno más estricto de lo que se ha llamado ${ }^{1}$, «teoría de los derechos», es decir, el plano analítico, mientras el problema de la fundamentación aparecería como cuestión estrictamente filosófica (según algunos, en realidad, metajurídica) y por ello objeto de estudio de la «Filosofía de los derechos» o, en todo caso, de lo que la tradición anglosajona considera Jurisprudencia censoria» o normativa. Una posición que, llevada al extremo, conduce a adoptar, lisa y llanamente, un punto de vista escéptico sobre ese modo de abordar la cuestión que lleva a calificar todo esfuerzo de fundamentación como «una empresa desesperada» ${ }^{2}$. Según esto, sería posible discutir sobre el problema conceptual, sobre la posibilidad y tipos de definición de «derechos» y sobre las dificultades terminológicas que todo ello encierra, sin necesidad de plantearse la cuestión de su fundamento, o, mejor, manteniendo

${ }^{1}$ Cfr. p. ej. Peces-Barba, 1983, donde insiste en la diferenciación entre Teoría de los derechos fundamentales y Filosofía de los derechos fundamentales que ya se encuentra en su clásico trabajo de 1978. Véase asimismo su toma de posición acerca del problema de la relación entre historicidad y concepto de derechos en Peces-Barba 1987 y de nuevo sobre la fundamentación en Peces-Barba 1989, donde reproduce la idea de la libertad igualitaria como clave del fundamento -desde luego, ético- de unos derechos de los que no cabe hablar sino refiriéndose a una «realidad integrable en el Derecho Positivo».

2 Bobbio, 1988. Esta posición de Bobbio es quizá el paradigma del punto de vista positivista/normativista sobre el problema. En cierto modo, puede ser calificada, con Bulygin(1987), como una toma de posición «realista»: se trataría de reconocer que en este terreno no hay apoyo sólido y que la pretensión contraria es incluso, «políticamente sospechosa, pues tiende a crear una falsa sensación de seguridad». 
esto último extramuros en una, en mi opinión, más que dudosa fortaleza conceptual.

Considero que dicho punto de partida entraña algunos riesgos entre los que no es el menor el de contribuir a mantener una ausencia de distinción entre los conceptos de derechos humanos (o «derechos» sin más) y derechos fundamentales, al que podemos añadir el peligro de pensar que se puede ofrecer un concepto de derechos que pudieran compartir todas las posiciones doctrinales que tratan de dar cuenta de la fundamentación, con independencia de su posición al respecto.

Sin duda, el ejemplo más claro de esta postura que sostiene la inexistencia de conexión necesaria entre ambos problemas y por ello la posibilidad de un tratamiento independiente de una y otra cuestión, concepción que en cierto sentido podríamos considerar característica de un acercamiento «analítico» al problema, es la teoría dualista ${ }^{3}$, tal y como la viene proponiendo entre nosotros el profesor Peces-Barba, desde luego no sin matizaciones: en efecto, elegir esta concepción es, para Peces-Barba, sobre todo un criterio metodológico, detrás del cual pueden aparecer muy diferentes respuestas conceptuales al problema de los derechos y no menos diferentes propuestas de respuesta a la interrogante relativa a la justificación o fundamentación de los mismos, pero lo que constituye el núcleo de esa posición es su rechazo tanto de las concepciones que ofrecen una respuesta monista a los dos problemas de concepto y fundamentación como a las que descartan ocuparse de ello por considerarlo una tarea estéril. En particular, la concepción

${ }^{3}$ Sobre la definición de la posición «dualista», caracterizada en principio como un criterio metodológico, resulta necesario hacer alguna precisión. Efectivamente, hay que distinguir de un lado la concepción dualista estrictamente metodológica que se limita (aunque no es poco) a poner de relieve la necesidad de distinguir la dimensión jurídica -inevitablemente jurídicopositiva- de los derechos humanos, de las posibilidades de justificación de los mismos, que no son sólo (aunque sí primordialmente) de carácter ético y, de otro lado, lo que podríamos calificar como «dualismo ontológico», el que critica Vernengo cuando se refiere a las tesis de quienes sostienen la concepción de los derechos morales (Vernengo 1989, pp. 327 y ss., praes. 340/42). Respecto a este último, creo que Vernengo tiene razón al denunciar la prolongación del dualismo metafísico de raigambre platónica en autores que tratan de mantener esa ambivalencia de los derechos: a la par, instituciones jurídicopositivas «imperfectas» y valores universales, sobre todo por cuanto de ese modo, en realidad lo que aparece es la tesis clásica de la concepción iusnaturalista de los derechos humanos. Sin embargo, parece claro que no todos los defensores de la teoría de los derechos morales son acreedores de esa etiqueta de dualismo ontológico y, por consiguiente, de iusnaturalismo. Explícitamente Vernengo reconoce que no sería así en el caso de la construcción de Laporta, y desde luego E. Fernández y A. Ruiz Miguel rechazan expresamente, por su parte, las razones de tal calificación. 
dualista se sitúa en manifiesta oposición (así el propio Peces-Barba o Prieto Sanchís) a aquellas tesis doctrinales que nos proponen la noción de «derechos morales» (concepto éste que, en el sentir de tales críticas ${ }^{4}$, tendría todos los inconvenientes del iusnaturalismo, sin aportar ninguna novedosa ventaja).

Por su parte, Pérez Luño ${ }^{5}$, separando también las cuestiones de concepto y fundamentación: por esa razón, propone a su vez (a) una fundamentación de carácter iusnaturalista, pues «los derechos humanos responden a instancias o valores éticos anteriores al derecho positivo», y en ese sentido muestra su cercanía a las tesis de los «derechos morales» que, según argumenta convincentemente, serían sobre todo «una denominación nueva para aludir a las exigencias éticas implícitas en la noción de los derechos humanos tal y como tradicionalmente han sido entendidos desde la óptica iusnaturalista», y por ello, encuentra equiparables las nociones de «derechos humanos», «derechos naturales» y «derechos morales». Al mismo tiempo, ofrece (b) un concepto de los derechos diversos de esta fundamentación: los derechos humanos serían «una categoría histórica que tan sólo puede ser predicada con sentido en un contexto determinado ${ }^{6} »$, o, como los define en otro lugar, un conjunto de facultades e instituciones que, en cada momento histórico, concretan unas exigencias o valores fundamentales ${ }^{7}$. Por tanto, aunque pueda parecer sorprendente, considero que de esa forma las tesis de la mencionada concepción dualista de Peces-Barba (fundamentación ética, que combina con un concepto histórico de los derechos humanos) convergen no poco con las de Pérez Luño, (concepto histórico de los derechos y fundamentación próxima al iusnaturalismo, si bien es cierto que muy matizadamente), aún partiendo de diferentes posiciones. En cualquier caso, convendría dejar señaladas ya, como lo hace entre otros Laporta, algunas observaciones elementales sobre el lugar de la historia en la justificación de los derechos: (a) Es evidente que el recurso a la Historia resulta imprescindible para explicar la génesis del desarrollo de los derechos humanos, para comprender de qué habla-

${ }^{4}$ Así, Peces-Barba 1987 y 1989, o Prieto Sanchís 1988 y 1989, donde se reiteran los argumentos contra las tesis sostenidas entre nosotros por E. Fernández $(1984,1987,1989)$ y que aparecen también tras el riguroso e interesante análisis de Laporta (1987). Vid. también Laporta 1989 y Ruiz Miguel 1989).

${ }^{5}$ Pérez-Luño 1984 y 1987. Las citas textuales corresponden a este último trabajo.

${ }^{6}$ Pérez Luño 1987.

${ }^{7}$ Cfr. Pérez Luño 1984, p. 51. 
mos cuando nos referimos en cada momento a los derechos, por qué y cuándo aparecen: y ello no significa necesariamente, como se ha indicado con acierto ${ }^{8}$, que se mantenga una concepción lineal de la Historia, sino sólo, entre otras consecuencias de interés, que, por ejemplo, pueden surgir nuevos derechos en respuesta a nuevas necesidades. (b) Asimismo, parece claro que el concepto de derechos humanos pertenece -como tantos otros: el de Estado- a la categoría de los conceptos históricos, con lo que todo ello implica: por ejemplo, que sólo tiene sentido hablar de ellos desde lo que se ha llamado (Peces-Barba) el «tránsito a la modernidad» y que, como escribe Pérez Luño, «ese contexto genético otorga... unos perfiles ideológicos definidos». (c) Finalmente, y en punto a la argumentación dirigida a proporcionar justificación o fundamento de los derechos, la Historia no puede ser razón justificativa, como argumenta ejemplarmente el mismo Laporta9: en primer lugar, «la validez de los enunciados se determina por argumentación racional... el hecho de que se presenten en un momento histórico no añade nada a esa argumentación»; además, resulta difícil cohonestar postulados morales y concepciones necesarias de la historia -pesimistas u optimistas-; finalmente, la historia no es un criterio, sino un totum revolutum, respecto al cual no cabe más que un conocimiento ex post. Por ello no cabe sino descartar (y así lo hacen Peces-Barba ${ }^{10}$ y Pérez Luño ${ }^{11}$ ) la fundamentación «historicista».

\section{II}

Seguramente la concepción dualista se nos muestra como la más útil, en el caso de que se pudiera solventar el problema que he tratado de destacar más arriba, como intentaré justificar después. Sin embargo, y a los efectos de mi argumentación, lo que me parece más interesante es mostrar cómo se plantea la cuestión de la relación entre concepto y fundamento de los derechos en

${ }^{8}$ Pérez Luño 1987, p. 56.

${ }^{9}$ Laporta 1987, pp. 75-76.

${ }^{10}$ Peces-Barba 1987, p. 220, que prefiere hablar de «fundamentación positivista», aunque, en realidad, acepta la fundamentación ética con tal de mantener que en ese plano no cabe hablar de derechos, sino de valores morales: sólo cuando esa moralidad se incorpora al derecho positivo podría hablarse de derechos fundamentales.

${ }^{11}$ Difícilmente podría ser de otra manera en quien entiende que la positivación de los derechos es un acto declarativo y no constitutivo: cfr. Pérez Luño, 1984; 1987). 
algunos de los representantes más significativos de la mencionada teoría de los «derechos morales», entre los que me referiré, por seguir en el contexto español, a las tesis de E. Fernández y F. Laporta. Efectivamente, como indiqué antes, tal teoría parece proporcionar una respuesta que evita la artificial separación entre las cuestiones de concepto y fundamentación de los derechos humanos. Sin embargo, en mi opinión, nos proporciona un ejemplo relevante de cómo se nos propone primordialmente como una respuesta dirigida aparentemente al problema conceptual, relativa al qué son los «derechos humanos» y por tanto distinta del problema de la justificación (aunque siempre en perfecta coherencia con ella), cuando en realidad es una teoría sólo acerca de esta segunda cuestión y no de la primera. En mi opinión, E. Fernández advierte bien el problema cuando escribe que el suyo es un intento de reunir fundamentación ética de los derechos humanos y concepto de los mismos como derechos morales. El inconveniente es que no se comprende bien qué hay de concepto y qué de fundamentación, precisamente porque el calificativo de «morales», según E. Fernández, trata de mostrar que el fundamento de los mismos es un fundamento ético, previo a lo jurídico (fundamento ético que, en realidad, como admite el propio autor, remite a las necesidades) de donde podríamos deducir que la expresión «derechos morales» no supondría en realidad una respuesta al problema del concepto. A ello responde E. Fernández ${ }^{12}$ con un argumento de gran interés, que podemos encontrar en otros defensores de los «moral rights» como Nino, Laporta, o Ruíz Miguel ${ }^{13}$ : si nos planteamos qué quiere decir la afirmación «tengo derecho a X», parece posible contestar que esa expresión no se reduce a ámbitos jurídicos y que, hablando con propiedad, «tiene un claro e irrenunciable origen y componente moral». Por esa razón niegan que la expresión tenga carácter confundente: los derechos humanos «son tales por su carácter moral, siendo accidental el reconocimiento jurídico para su concepto», y, por lo demás, desde el punto de vista pragmático de la defensa de los derechos ésta les parece la posición más eficaz ${ }^{14}$. Sin embargo, el problema se mantiene en tanto que los partidarios de la teoría de los derechos morales adopten el punto de vista estrictamente analítico en aras del rigor científico, reconociendo que desde las exigencias de la lógica resulta posible admitir que la cuestión

\footnotetext{
${ }^{12}$ Ibid., p. 158;

${ }^{13}$ Cfr. Fernández 1989, p, 158. Vid. también Páramo 1987, Laporta 1987 y Ruiz Miguel 1989.

${ }^{14}$ Ruiz Miguel 1989, pp. 322 y 325.
} 
conceptual es un problema previo y, por consiguiente, centrándose aparentemente en ese único tema, todo ello aunque expresamente se advierta a menudo (si bien luego no se sepa o se pueda respetar tal exigencia), que ese trabajo de deslinde, por más que sea útil y aún imprescindible, se revela un auténtico castigo de Sísifo por las dificultades que conlleva: estamos aquí, una vez más, ante un problema circular, aunque con ello no quiero decir que se trate tan sólo de un problema terminológico en el sentido impreciso (y por ello peyorativo) de la palabra, puesto que las consecuencias de utilizar una u otra acepción son de cierta importancia práctica (sería un problema semántico en el sentido estricto de ese término).

En definitiva, el problema podría describirse en estos términos: cuando quienes se ocupan de la cuestión conceptual tratan de responder a la pregunta ¿qué significa tener derecho a $\mathrm{X}$ ? ofreciendo la noción de derechos morales, casi nunca ofrecen una respuesta en el plano conceptual, sino que las más de las veces formulan propuestas que deberían situarse en el de la justificación, es decir, proporcionan una tesis fundamentadora de los derechos, y no un concepto de derechos en cuanto tal. Trataré de explicarlo aludiendo precisamente a la construcción de Laporta que me parece uno de los intentos más acabados de evitar esa confusión aún reconociendo lo esencial de la teoría de los derechos morales.

El trabajo de F. Laporta ${ }^{15}$, que constituye un punto inevitable de referencia, comienza precisamente por poner de relieve la dificultad de delimitación de los problemas de concepto y fundamentación, pero en definitiva, y en aras del rigor científico, adopta una consideración específica del problema conceptual para el que propone como respuesta la noción de derechos morales. Su reflexión sobre el concepto de derechos humanos como moral rights, se apoya, entre otros argumentos básicos, en la distinción (en línea con la crítica de MacCormick a buena parte de los planteamientos habituales) entre «derechos» $\mathrm{y}$ «técnicos de protección»: los derechos serían, por expresarlo de alguna manera, entidades «prenormativas», mientras que las facultades de ejecución o renuncia a los derechos, los «poderes» atribuidos por el Derecho positivo, existirían sólo después de que existan los

${ }^{15}$ Me referiré, salvo mención expresa, a Laporta 1987. Con posterioridad (Laporta 1989) ha vuelto sobre el tema para precisar el sentido y alcance de los esfuerzos de fundamentación de los derechos, a propósito de lo cual recuerda la necesidad de separar los planos de la reflexión sobre derechos humanos y la relativa a los derechos fundamentales. Como se verá más adelante, habrá oportunidad de comentar este segundo trabajo. 
derechos, no serían el elemento constitutivo de los mismos. El punto de partida puede encontrarse en la tesis que señala - la cita es del propio Laporta- que en todo sistema normativo «no sólo pueden darse relaciones lógico-deductivas sino que aparecen también relaciones justificatorias o instrumentales que pertenecen al mundo del razonamiento práctico y son imposibles de aprender en los lazos formales del razonamiento lógico-deductivo» ${ }^{16}$. Por mi parte, trataré de mostrar cómo, precisamente, la respuesta de los «moral rights» esconde, tras su equívoco, la pista certera, como apuntan, entre otros, Atienza y Ruiz Manero cuando en su comentario de las tesis de Laporta escriben: «... a su concepción de los derechos como entidades pre-normativas podría acusársele de confundir los derechos con el fundamento de los mismos $\gg{ }^{17}$. Efectivamente, Laporta sale al paso de esta crítica a su propia concepción indicando que quien la formula ignora una precisión básica: para él los derechos (morales) son entidades prenormativas pero que forman parte del sistema jurídico, concretamente como «razones intrasistemáticas» (razones que forman parte del sistema jurídico y que sirven como argumento para la existencia de los derechos legales), mientras que el fundamento de los derechos (legales) sería siempre un problema de «justificación extrasistemática» de los mismos. En el fondo, siempre según Laporta, el problema derivaría del prejuicio «legalista» que radica en entender que sólo tiene sentido la expresión «tener un derecho» cuando existe una técnica de protección que consiste en poner en manos del titular un dispositivo institucional de coacción lo que, sin embargo, no sería sostenible desde un punto de vista empírico ni aún para los sistemas que llamamos jurídicos. Por lo demás, se trataría de constatar simplemente que al igual que no hay correlación necesaria entre obligaciones morales y obligaciones legales o jurídicas, tampoco la hay entre derechos morales y derechos legales, afirmación de la que derivan dos consecuencias del mayor interés:

a) Sostener la tesis de los derechos morales no significaría necesariamente adoptar posiciones iusnaturalistas: no es así, desde luego, en la construcción de Laporta: no se trata ni de la existencia de «otro» orden jurídico, ni de la tesis de la necesaria intersección ente Derecho y Moral que es uno de los puntos básicos de la doctrina del Derecho Natural ${ }^{18}$.

\footnotetext{
${ }^{16}$ Laporta, 1987, p. 29.

${ }^{17}$ Atienza-Ruiz Manero 1987, p. 69.

${ }^{18}$ En el mismo sentido, cfr. las afirmaciones de E. Fernández 1989 y A. Ruiz Miguel 1989.
} 
b) Sostener la tesis de los derechos morales significaría sólo (pero no es poco) que junto al orden jurídico empírico existe un orden moral y que a uno y otro corresponden derechos: lo que llamamos habitualmente derechos humanos pertenecerían a este ámbito de la moralidad: serían derechos morales, o, lo que es lo mismo, violarlos sería violar derechos y por eso su desconocimiento «justifica acciones como la desobediencia a las leyes y la resistencia a la opresión jurídico-positiva».

Pues bien, entiendo que hay una contradicción entre argumentar que los derechos (morales) son elementos intrasistemáticos del sistema normativo jurídico y afirmar que, al igual que las obligaciones morales, pertenecen al ámbito de la moralidad y no tienen una necesaria correlación en derechos legales, esto es, en el sistema normativo. Esto es: decir que los derechos (morales) son algo que está «más allá de las normas... constituyen la razón para articular una protección mediante normas» significa, en mi opinión, que la noción de derechos morales no pertenece al sistema normativo jurídico hasta tanto los derechos morales no son incorporados al sistema normativo jurídico como razones que justifican la existencia de los derechos legales, pero debe advertirse que esa incorporación no es (1) necesaria (los derechos morales pueden continuar en el ámbito exclusivamente moral, como razones para la acción) y por consiguiente (2) no es razón suficiente que nos permita calificar a los derechos morales, en definitiva, no son tales derechos, sino que ofrecen razones para justificar la exigencia de un reconocimiento normativo determinado: para fundamentar los derechos (los derechos fundamentales) ${ }^{19}$.

\section{(III)}

En otras palabras: lo que sucede es que los derechos morales serían como ha visto bien Laporta, «razones para la acción», especialmente pertinentes en el ámbito de la praxis moral, jurídica y política, es decir, fundamentan juicios de justicia o legitimidad y/o apoyan la adopción de respuestas concretas para materializar esas exigencias (esa es la verdad que encierra el tópico tan

${ }^{19}$ En otras palabras: el problema es el mismo que plantea la innovación de las necesidades como derechos, mejor, como fundamento de los mismos, según hemos tratado de mostrar en De Lucas/Añón, 1990, aunque con ventaja para el recurso a las necesidades. 
repetido de que los derechos humanos constituyen la concreción de la idea de justicia en nuestra conciencia colectiva, en nuestro horizonte cultural), porque, como advierte el propio Laporta en otro lugar, «si los enunciados jurídicos que reconocen u otorgan derechos humanos son vinculantes ello se debe a que son conclusiones de un razonamiento práctico cuyas premisas prácticas o valorativas son extrajurídicas ${ }^{20}$. Eso es tanto como reconocer que el lugar de la teoría de los derechos morales en relación con los derechos humanos es el de la justificación o fundamento de los derechos (tanto si nos referimos a los derechos humanos en general, como, a fortiori, si se trata de los derechos fundamentales), y no constituyen una respuesta al problema conceptual, a la pregunta ¿qué son los derechos?, sino a la pregunta ¿por qué debe aceptarse que tengo un derecho? o, mejor, ¿por qué está justificado exigir del Derecho una respuesta normativa? Por lo demás, si se me permite la expresión, en realidad la teoría de los derechos morales ni siquiera ofrece una respuesta al problema de la justificación, pues constituye un circunloquio, un rodeo que remite, en la mayoría de los casos, bien a una respuesta antropológica iusnaturalista, bien a una axiología, bien, lo que quizá es hoy la posición más interesante, a una variante de la solución «antropológica» basada en la noción de las necesidades humanas. Efectivamente, como se ha indicado por Añón ${ }^{21}$ entre otros, serían las necesidades, y no los derechos morales lo que proporcionaría, en todo caso, las razones, los argumentos que apoyarían una respuesta jurídico normativa (y no otra) a determinadas exigencias que se pretenden justificadas, una respuesta jurídica normativa a las demandas que afirman que es justo reclamar algo, una justificación de por qué tiene fundamento racional pretender que el Derecho adopte -en cuanto sistema normativo y con las técnicas que ello supone- una respuesta a tales demandas: una respuesta, pues, que no consiste tan sólo en

${ }^{20}$ Laporta 1989, p. 205. En este trabajo, en el que se realiza un brillante análisis acerca de qué tipo de enunciados morales pueden proporcionar fundamentación de enunciados jurídicos y, más concretamente, derechos fundamentales, lo que entre otras cosas supone una precisión del carácter vinculante de los enunciados jurídicos relativos a derechos fundamentales así como una distinción entre enunciados morales que sirvan para el objetivo de fundamentación/justifícación, hay una premisa que no conviene olvidar, y es que, como advierte el autor, se está hablando de derechos fundamentales, dentro del orden jurídico, y no de derechos humanos en general. Es decir: el recurso final a los derechos morales es un recurso para ofrecer un fundamento de los derechos positivos.

${ }^{21}$ Añón, 1990. 
técnicas de protección (normas primarias). Por esa razón, la propuesta de los derechos morales como concepto de derechos humanos no es, en mi opinión, operativa: no ofrece más de lo que podemos conseguir por otras vías que, sin embargo, evitan una confusión que no sólo es conceptual, sino también metodológica. Otra cosa sería afirmar que en orden a proporcionar las relaciones de justificación propias del ámbito del razonamiento práctico que son imprescindibles para cualquier intento de fundamentación, el recurso a la noción de derechos morales pueda tener, como en mi opinión la tiene, una gran utilidad, sin que ello comporte que los derechos morales sean el auténtico fundamento: en realidad tal fundamento está más allá, y nos lo proporcionarían criterios como los de bienes básicos o necesidades a los que precisamente remite la noción de derechos morales, entre otras razones por las inequívocas limitaciones de la propia teoría de los derechos morales ${ }^{22}$.

\section{BIBLIOGRAFÍA}

Atienza/Ruíz Manero (1987), «A propósito del concepto de derechos humanos de F. Laporta», Doxa, 4.

Añón (1991), «Fundamentación de los derechos humanos, y necesidades básicas», en Ballesteros (ed.) y varios autores, Los derechos humanos, Madrid, Tecnos (en prensa).

Ballesteros/ De Lucas (1989), «Sobre los límites del principio de disidencia», en Muguerza y varios autores, El fundamento de los derechos humanos, Madrid, Debate.

Bobbio (1988), El problema de la guerra y las vías para la paz, Madrid, Gedisa.

Bulygin (1987), «Sobre el estatuto ontológico de los derechos humanos», Doxa, 4.

Fernández (1984), Teoría de la justicia y derechos humanos, Madrid, Debate.

Fernández (1989), «Acotaciones de un supuesto iusnaturalista a las hipótesis de J. Muguerza sobre los derechos humanos», en Muguerza 1989 cit.

22 En este sentido recojo la crítica de Prieto Sanchís 1987 a la dimensión liberal-individualista la teoría de los derechos morales. Efectivamente, como sostiene Ruiz Miguel en sintonía con las tesis de Muguerza (Muguerza 1989), resulta difícil dejar de admitir que la moralidad tiene su raíz en el individuo que es llamado a disentir, en la moral crítica o moral abierta, como se prefiera, pero creo que eso no resuelve la objeción del lastre ideológico denunciado en la teoría de los derechos morales, y así lo hemos tratado de argumentar en otro lugar (Ballesteros-De Lucas 1989). 
Laporta (1987), «Sobre el concepto de derechos humanos», Doxa, 4.

Laporta (1989), «Sobre la fundamentación de enunciados jurídicos de los derechos humanos», en Muguerza 1989 cit.

De Lucas/Añón (1989), «Necesidades, razones, derechos», Doxa, 7.

Muguerza (1989), «La alternativa del disenso», en Muguerza, El fundamento de los derechos humanos, Madrid, Debate.

Nino (1989), Ética y derechos humanos, Barcelona, Ariel.

Nino (1990), «Sobre los derechos morales», en Doxa, 7.

Páramo (1989), «Bienestar, derechos y autonomía», en Muguerza 1989 cit.

Peces-Barba (1983), Derechos fundamentales, Madrid, Univ. Comp.

Peces-Barba (1987), «Sobre el puesto de la Historia en los derechos fundamentales», Anuario de Derechos Humanos, 4.

Peces-Barba (1988), «Sobre el fundamento de los derechos humanos», en Muguerza 1989, cit.

Pérez Luño (1984), Derechos Humanos, Estado de Derecho y Constitución, Madrid, Tecnos (hay 2. a ed. 1986).

Pérez Luño (1989), «Sobre los valores fundamentadores de los derechos humanos», en Muguerza 1989 cit.

Pérez Luño (1988), Los derechos fundamentales, Madrid, Tecnos.

Prieto Sanchís (1987), «Ideología liberal y fundamentación iusnaturalista de los derechos humanos. Observaciones críticas», Anuario de Derechos Humanos, 4.

Ruiz Miguel (1989), «Los derechos humanos como derechos morales: entre el problema verbal y la modernización confusa», en Muguerza 1989, cit.

Ruiz Miguel (1990), «Los derechos humanos como derechos morales», Anuario de Derechos Humanos, 6.

Vernengo (1989), «Los derechos humanos y sus fundamentos éticos», en Muguerza 1989 cit.

Vernengo (1990), «Enfoques escépticos de los derechos humanos», Anuario de Derechos Humanos, 6. 